Pflege in der Klinik

\title{
Personaluntergrenzen kommen
}

— Für besonders pflegeintensive Bereiche im Krankenhaus sollen künftig Pflegepersonaluntergrenzen festgelegt werden, z.B. für Intensivstationen und Nachtdienst. Darauf haben sich Bundesgesundheitsministerium, Koalitionsfraktionen und Bundesländer geeinigt.

Die Ergebnisse der Beratungen in der Expertenkommission „Pflegepersonal im Krankenhaus" sehen vor, die Selbstverwaltung von Krankenhäusern und Krankenkassen gesetzlich zur Vereinbarung von Personaluntergrenzen zu verpflichten. Die Vereinbarung soll bis zum 30. Juni 2018 getroffen und zum 1. Januar 2019 wirksam werden. Sollte bis zum 30. Juni 2018 keine Vereinbarung der Selbstverwaltung zustande kommen, wird das Bundesministerium für Gesundheit bis zum 31. Dezember 2018 ersatzweise die ausstehenden Entscheidungen treffen. „Eine gute Versorgung im Krankenhaus setzt eine angemessene Personalaus-

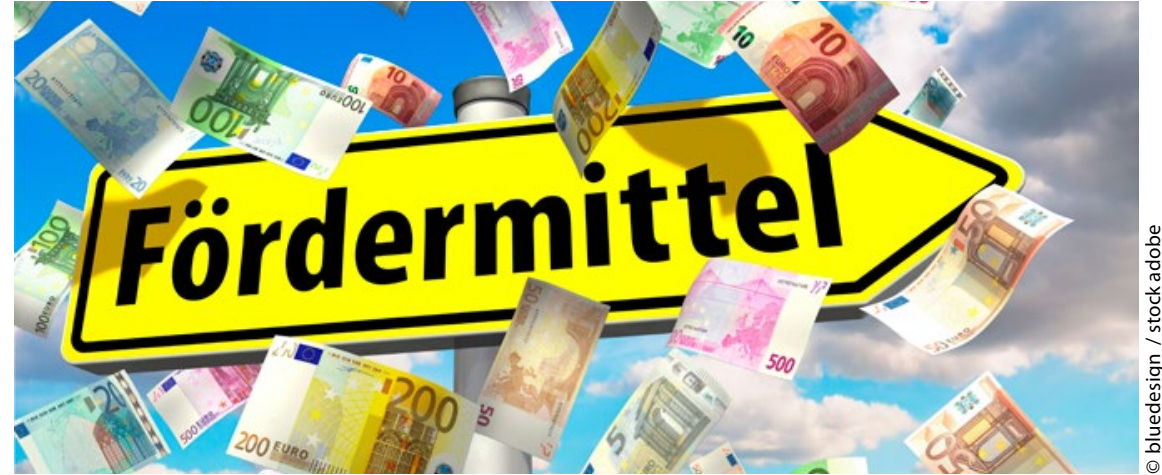

stattung voraus", erklärt Bundesgesundheitsminister Hermann Gröhe. Jetzt müsse es darum gehen, dass die kommenden Regelungen fristgerecht von den Krankenhäusern und Krankenkassen mit Leben gefüllt würden. Um dauerhaft mehr Personal beschäftigen zu können, werden die Krankenhäuser seit diesem Jahr durch einen Pflegezuschlag unterstützt. Dieser soll ab 2019 um die Mittel des Pflegestellen-Förderprogramms ergänzt werden und damit von bisher 500 Millionen Euro auf bis zu 830 Millionen Euro pro Jahr anwachsen.

Der DBfK begrüßt die Ergebnisse der Kommission. Der Verband fordert, bei der Umsetzung der Pläne - insbesondere bei der
Definition von "sensitiven Bereichen“ - daverteilung von Planstellen aus einem Bereich in den anderen kommt. Die pflegerische Versorgung insgesamt müsse spürbar verbessert werden. Die festzulegenden Personaluntergrenzen müssen laut DBfK die Personalausstattung definieren, die für die Patientensicherheit unabdingbar ist. Sie ersetzen nicht eine Personalbemessung, die sich am tatsächlichen Versorgungsbedarf orientiert, heißt es weiter. Das werde eine wichtige Aufgabe für die nächste Legislaturperiode sein.

www.bundesgesundheitsministerium.de für Sorge zu tragen, dass es nicht zur Um-

\section{Aktionstag}

\section{Bundesweite "Gefährdungsanzeige" soll Politik wachrütteln}

— Für den 12. Mai hat die Initiative "Pflege in Bewegung" zu einem Aktionstag in Berlin aufgerufen. Der Verein hat bundesweite "Gefährdungsanzeigen" aufgesetzt, die Personalmangel, inakzeptable Arbeitsbedingungen und miese Entlohnung der beruflich Pflegenden anprangern. Kritisiert werden auch die schlechten Rahmenbedingungen für pflegende Angehörige. Unterstützer können Flyer unterschreiben und an den Verein senden. Die Unterschriften sollen am Aktionstag Bundesgesundheitsminister Hermann Gröhe übergeben werden. „Auf Grund des demographischen Wandels und des sich verschärfenden Pflegenotstandes ist es an der Zeit, die Politik zum sofortigen Handeln zu bewegen", so Roger Konrad, Vorsitzender von "Pflege in Bewegung".

Unterstützt wird die Initiative unter anderem von der rheinland-pfälzischen Pflegekammer. Präsident Dr. Markus Mai: „Wir sind stolz, Unterstützer und Wegbegleiter dieser jungen Bewegung von professionell Pflegenden und pflegenden Angehörigen zu sein." Deshalb unterstütze man die Kampagne zur Gefährdungsanzeige, die in weiten Teilen zu den kammereigenen Forderungen passe. Weitere Wegbegleiter sind einige Städte des bundesweiten Personenbündnisses „Pflege am Boden“ und die Siegener Initiative „Wa(h)re Gesundheit - da hilft nur noch beten?".

www.pflegeinbewegung.de

Vorschau!

Die PflegeKollegs im Monat Mai:

Gestörte Wundheilung

Biofilm - Wundauflagen

- Patientenedukation

Alternativ pflegen

Tai-Chi und Qi Gong - Anthroposophische Pflege - Therapeutic Touch 